\title{
Electrolyte effect on the phase behavior of silica nanoparticles with lysozyme and bovine-serum-albumin proteins
}

\author{
Indresh Yadav, ${ }^{1}$ V. K. Aswal, ${ }^{1}$ and J. Kohlbrecher ${ }^{2}$ \\ ${ }^{1}$ Solid State Physics Division, Bhabha Atomic Research Centre, Mumbai 400 085, India \\ ${ }^{2}$ Laboratory for Neutron Scattering, Paul Scherrer Institut, CH-5232 PSI Villigen, Switzerland
}

(Received 24 December 2014; published 29 May 2015)

\begin{abstract}
Small-angle neutron scattering (SANS) and dynamic light scattering (DLS) studies have been carried out to investigate the effect of an electrolyte on the phase behavior of anionic silica nanoparticles with two globular proteins-cationic lysozyme [molecular weight (MW) $14.7 \mathrm{kDa}$ ] and anionic bovine serum albumin (MW $66.4 \mathrm{kDa}$ ). The results are compared with our earlier published work on similar systems without any electrolyte [I. Yadav, S. Kumar, V. K. Aswal, and J. Kohlbrecher, Phys. Rev. E 89, 032304 (2014)]. Both the nanoparticleprotein systems transform to two phase at lower concentration of protein in the presence of an electrolyte. The autocorrelation function in DLS suggests that the diffusion coefficient $(D)$ of a nanoparticle-protein system decreases in approaching two phase with the increase in protein concentration. This variation in $D$ can be attributed to increase in attractive interaction and/or overall increase in the size. Further, these two contributions (interaction and structure) are determined from the SANS data. The changes in the phase behavior of nanoparticle-protein systems in the presence of an electrolyte are explained in terms of modifications in both the repulsive and attractive components of interaction between nanoparticles. In a two-phase system individual silica nanoparticles coexist along with their fractal aggregates.
\end{abstract}

DOI: 10.1103/PhysRevE.91.052306

PACS number(s): 82.70.Dd, 87.14.E-, 61.46.-w, 61.05.fg

\section{INTRODUCTION}

The conjugation of nanoparticles to biomolecules (e.g., lipid, protein, and DNA) has attracted significant interest in recent years in the area of nanobiotechnology [1,2]. In particular, the nanoparticle-protein conjugates find applications in drug delivery, protein crystallization, biological imaging, novel functional materials, etc. [3-6]. Therefore, the interactions of nanoparticles with different proteins are most studied. Understanding these interactions establishes the predictive relationship between the structure and functionality, which depends on the intrinsic characteristics (e.g., sizes, shapes, and charges) of both the nanoparticles and proteins $[7,8]$. There is a strong propensity of protein to adsorb on the nanoparticles due to the high surface-to-volume ratio of the nanoparticles. The formation of a corona around each nanoparticle is determined by the different interactions (e.g., electrostatic force, hydrogen bonding, etc.) between nanoparticle and protein [7,9,10]. However, the resultant structure (phase behavior) depends on the nanoparticle-protein, nanoparticle-nanoparticle, and protein-protein interactions. The phase behavior for a given nanoparticle-protein system can be modulated by changing the solution conditions such as the concentrations of components, $p \mathrm{H}$, ionic strength, etc. [7,11,12].

The Derjaguin-Landau-Verwey-Overbeek (DLVO) theory has been successfully used to explain the phase behavior of a number of charged colloidal systems $[13,14]$. However, this theory has limitations in the case of nanoparticle-protein systems because of the presence of non-DLVO interactions (e.g., solvation, steric, and depletion) $[15,16]$. There have been studies of the phase behavior of charged stabilized silica nanoparticles with both similarly and oppositely charged proteins [17-19]. In the case of oppositely charged protein, the nanoparticle-protein system is two phase (nanoparticle aggregation) due to the protein-mediated aggregation of nanoparticles [18]. On the other hand, the possibility of site-specific adsorption of similarly charged protein on individual nanoparticles enhances the stability of the system [20,21]. In the absence of site-specific adsorption, the nonadsorption of protein can induce the depletion force and modify the phase behavior of the nanoparticle-protein system [22]. We have recently reported the similar phase behavior of anionic silica nanoparticles with cationic lysozyme and anionic bovine serum albumin (BSA), where both the proteins induce nanoparticle aggregation [23]. The aggregation of nanoparticles is interpreted as a result of domination of short-range attraction (protein mediated) for lysozyme and long-range attraction (protein depleted) for BSA over the long-range electrostatic repulsion between the nanoparticles. The resultant interaction is decided by the combination of different interactions between components (nanoparticle-protein, nanoparticle-nanoparticle, and protein-protein) present in the system [19]. In the present study, we have investigated the effect of an electrolyte modifying the phase behavior of nanoparticle-protein systems in terms of these interactions.

We have studied the phase behavior of anionic Ludox silica nanoparticles HS40 with cationic lysozyme [molecular weight (MW) $14.7 \mathrm{kDa}$ ] and anionic BSA (MW $66.4 \mathrm{kDa}$ ) at $p \mathrm{H} 7$ and in the presence of an electrolyte $(\mathrm{NaCl})$. The results with the electrolyte effect are compared with those of our earlier work without electrolyte [23]. The systems have been characterized by a combination of small-angle neutron scattering (SANS) and dynamic light scattering (DLS). SANS is an excellent technique to study both the interaction and structure in multicomponent systems [24,25]. It gives the scattering profile in Fourier space, and the real space information (structure and interaction) of the sample is determined by fitting the experimental data with suitable mathematical models [26]. The evolution of the phase behavior of the nanoparticle-protein system has been modeled using the two-Yukawa (2Y) potential accounting for the competitive electrostatic repulsion and attractive interaction. The $2 \mathrm{Y}$ 
potential provides the strength and the range of the individual parts (repulsive and attractive) of the total potential without any predefined assumption [27,28]. DLS is used as a technique complementary to SANS, where autocorrelation function provides the value of the diffusion coefficient, which can be related to the structure and interactions of the particles in the system [29,30].

\section{EXPERIMENTS}

Electrostatically stabilized colloidal suspension of $40 \mathrm{wt}$ \% of spherical silica nanoparticles (Ludox HS40) and proteins (hen egg lysozyme and BSA) were purchased from SigmaAldrich. Samples were prepared by dissolving the weighted amount of nanoparticles and proteins in $20 \mathrm{~m} M$ phosphate buffer at $p \mathrm{H} 7$ in $\mathrm{D}_{2} \mathrm{O}$. The choice of $\mathrm{D}_{2} \mathrm{O}$ as solvent instead of $\mathrm{H}_{2} \mathrm{O}$ in neutron scattering experiments provide high contrast and low incoherent background. All the measurements were carried out for fixed concentration of silica nanoparticles (1 wt. \%) with varying the concentration of proteins in the range $0-5$ wt. $\%$ and in the presence of $0.1 M \mathrm{NaCl}$. Small-angle neutron scattering experiments were performed on the SANS-I instrument at the Swiss spallation neutron source, SINQ, Paul Scherrer Institut, Switzerland [31]. The mean wavelength $(\lambda)$ of the incident neutron beam was $6 \AA$ with the wavelength resolution of approximately $10 \%$. The scattered neutrons were detected by using a $96 \times 96 \mathrm{~cm}^{2}$ detector. The experiments were performed at two sample-todetector distances of 2 and $8 \mathrm{~m}$, respectively, to cover the data in the scattering vector $[Q=4 \pi \sin (\theta / 2) / \lambda$, where $\theta$ is scattering angle] range of $0.006-0.25 \AA^{-1}$. The freshly prepared samples were held in Hellma quartz cells having thickness $2 \mathrm{~mm}$ and temperature kept constant at $30^{\circ} \mathrm{C}$ during the measurements. The data were corrected and normalized to absolute scale using standard procedure. DLS measurements were performed on nanoparticle size analyzer SZ-100 (Horiba, Japan). The wavelength of the incident laser light was $5320 \AA$ and the data were collected at scattering angle $173^{\circ}$.

\section{DATA ANALYSIS}

\section{A. Small-angle neutron scattering}

In small-angle neutron scattering, one measures the coherent differential scattering cross section per unit volume $(d \Sigma / d \Omega)$ as a function of scattering vector $Q$, and for a system of monodisperse particles it can be expressed as [32-34]

$$
\frac{d \Sigma}{d \Omega}(Q)=n V^{2}\left(\rho_{p}-\rho_{s}\right)^{2} P(Q) S(Q)+B,
$$

where $n$ is the number density and $V$ is the particle volume. $\rho_{\mathrm{p}}$ and $\rho_{\mathrm{s}}$ are scattering length densities of particles and solvent, respectively. $P(Q)$ is the intraparticle structure factor and depends on the shape and size of the particle. $S(Q)$ is the interparticle structure factor and is determined by the interaction between particles. $B$ is a constant term representing incoherent background.

For standard shapes of the particles, the analytical expressions for $P(Q)$ are available in the literature [24-26]. The interaction between particles may be attractive and/or repulsive and for such systems $S(Q)$ can calculated using the two-Yukawa potential (2Y) under mean spherical approximation [27,28]. The $2 \mathrm{Y}$ potential in terms of four dimensionless parameters $\left(K_{1}, K_{2}, Z_{1}\right.$, and $\left.Z_{2}\right)$ is given by [35]

$$
\frac{V(r)}{k_{B} T}=\infty(0<r<\sigma)=\frac{V_{2 Y}}{k_{B} T}(r>\sigma),
$$

where

$$
\begin{aligned}
\frac{V_{2 \mathrm{Y}}(r)}{k_{B} T}= & -K_{1} \frac{\exp \left[-Z_{1}(r / \sigma-1)\right]}{r / \sigma} \\
& +K_{2} \frac{\exp \left[-Z_{2}(r / \sigma-1)\right]}{r / \sigma},
\end{aligned}
$$

where $k_{B}$ is Boltzmann's constant, $T$ is absolute temperature, $r$ is the interparticle distance, and $\sigma$ is the hard sphere diameter of the particle.

The attractive interaction within the system could lead to the aggregates and be manifested by an increase of $S(Q)$ in the low- $Q$ region [36]. The scattering cross section for the particle aggregation can be expressed as [23]

$$
\frac{d \Sigma}{d \Omega}(Q)=n_{a} V_{p}^{2}\left(\rho_{p}-\rho_{s}\right)^{2} P(Q) S_{f}(Q)+B,
$$

where $n_{a}$ and $V_{p}$ are the number density and volume of individual scatter in the aggregates. $P(Q)$ is the intraparticle structure factor of the building block in aggregated structures. $S_{f}(Q)$ is the structure factor of the particle aggregates. The structure factor for a surface fractal is accounted for by the following equation $[37,38]$ :

$$
\begin{aligned}
S_{s f}(Q)= & Q^{-1} \Gamma\left(5-D_{s}\right) \xi^{5-D_{s}}\left[1+(Q \xi)^{2}\right]^{\left(D_{s}-5\right) / 2} \\
& \times \sin \left[\left(D_{s}-1\right) \tan ^{-1}(Q \xi)\right] .
\end{aligned}
$$

Equation (5) for $Q \gg 1 / \xi, S_{s f}(Q)$ simplifies to [37,38]

$$
S_{s f}(Q) \sim Q^{-\left(6-D_{s}\right)} \text {. }
$$

For the mass fractal, the structure factor is given as [39]

$$
\begin{aligned}
S_{m f}(Q)= & 1+\frac{1}{(Q R)^{D_{m}}} \frac{D_{m} \Gamma\left(D_{m}-1\right)}{\left[1+\frac{1}{(Q \xi)^{2}}\right]^{\left(D_{m}-1\right) / 2}} \\
& \times \sin \left\{\left(D_{m}-1\right) \tan ^{-1}(Q \xi)\right\} .
\end{aligned}
$$

Equation (7) for $Q \gg 1 / \xi, S_{m f}(Q)$ simplifies to [39]

$$
S_{m f}(Q) \sim Q^{-D_{m}}
$$

where $D_{s}$ and $D_{m}$ are surface fractal and mass fractal dimensions, respectively. $\xi$ is the maximum length up to which the fractal microstructure exists, $R$ is the size of building block, and $\Gamma$ is the mathematical gamma function.

The data have been analyzed by the nonlinear least-square fitting of the model scattering to the experimental data [40]. Throughout the analysis corrections were also made for instrumental smearing of the experimental data. The fitted data are given by the solid lines to the experimental data points.

\section{B. Dynamic light scattering}

DLS measures the intensity autocorrelation function of scattered light. Due to the Brownian motion of the suspended particles, interference between the scattered light produces a fluctuation in intensity as a function of time [41-43]. This 
temporal variation in intensity of light contains information about the motion of the particles. The normalized intensity autocorrelation function at a particular angle is given as [43]

$$
g^{(2)}(\tau)=\frac{\langle I(t) I(t+\tau)\rangle}{\left\langle I(t)^{2}\right\rangle},
$$

where $I(t)$ is the scattered light intensity at time $t$ and $I(t+\tau)$ the scattered light intensity at time $t$ plus a lag time $\tau$. For an ergodic system it is related to the field autocorrelation function by the Siegert relation as [43]

$$
g^{(2)}(\tau)=\beta\left[g^{(1)}(\tau)\right]^{2}+B,
$$

where $\beta$ is the spatial coherence factor which depends on the optical geometry and $B$ is the baseline.

For a monodisperse system of particles, $g^{(1)}(\tau)$ decay exponentially; i.e.,

$$
g^{(1)}(\tau)=\exp (-\Gamma \tau) .
$$

In the case of polydisperse particles, it can be written as [43]

$$
g^{(1)}(\tau)=\int_{0}^{\infty} G(\Gamma) \exp (-\Gamma \tau) d \Gamma,
$$

where $G(\Gamma)$ is the weight factor in the decay rate distribution. The average decay rate $\Gamma$ has been calculated using cumulant analysis [44] and is related to the diffusion coefficients $(D)$ of the particles and the magnitude of wave vector $q$ as follows:

$$
\Gamma=D q^{2} \text {. }
$$

The effective hydrodynamic size (diameter) of the particles is calculated from $D$ by the Stokes-Einstein relation as [41]

$$
d_{h}=\frac{k_{B} T}{3 \pi \eta D}
$$

where $k_{B}$ is Boltzmann's constant, $T$ is absolute temperature, and $\eta$ is the viscosity of the solvent.

\section{RESULTS AND DISCUSSION}

Figure 1 shows the phase behavior of 1 wt. \% HS40 silica nanoparticles with lysozyme and BSA proteins in the presence of $0.1 \mathrm{M} \mathrm{NaCl}$. The comparison is also made with already published phase behavior of nanoparticle-protein systems without any electrolyte [23]. The figure depicts the variation of transmission of light $(6000 \AA)$ through silica nanoparticles as a function of protein concentration. In each case the transmission of light decreases dramatically beyond a critical protein concentration (CPC). This point represents the outset of the nanoparticle-protein system transforming from one phase (clear) to two phase (turbid). It is noticed from Fig. 1 that the value of CPC strongly depends on the type of protein and the presence of an electrolyte. The CPC values are very different for the two proteins, which are expected because of their different interactions with the nanoparticles. It is observed that the phase behavior of silica nanoparticles with both the proteins is significantly modified in the presence of an electrolyte. The CPC values are suppressed on addition of an electrolyte. The effect is more pronounced for BSA than lysozyme. The evolutions of interaction and structure responsible for the electrolyte effect in phase behavior of

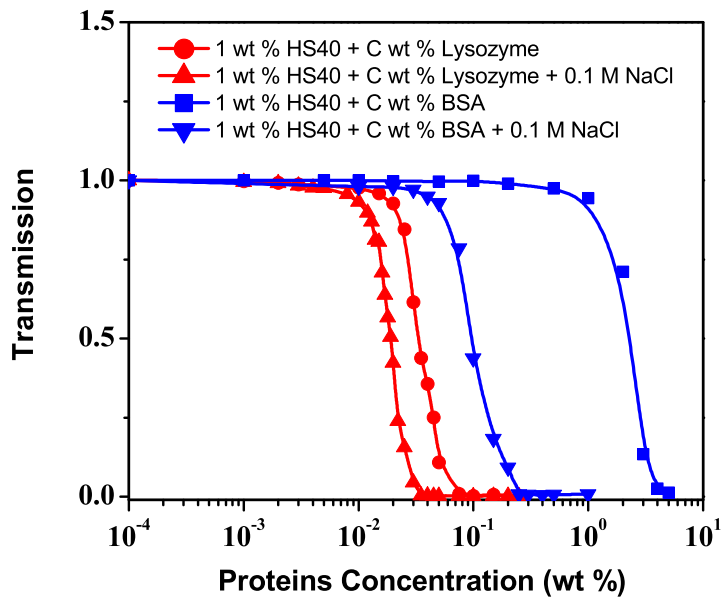

FIG. 1. (Color online) Transmission of light in $1 \mathrm{wt} \%$ HS40 silica nanoparticles with varying concentrations of lysozyme and BSA proteins in the presence of $0.1 \mathrm{M} \mathrm{NaCl}$. The results of nanoparticleprotein systems without any electrolyte from Ref. [23] are also shown.

nanoparticle-protein systems have been studied by SANS and DLS.

The DLS data of the silica nanoparticle-lysozyme system are shown in Fig. 2. The autocorrelation function is found to broaden with the increasing lysozyme concentration as approaching from a one-phase to a two-phase system. This implies protein-dependent decrease in the decay rate of the autocorrelation function and hence the decrease in the average diffusion coefficient. The variation of calculated diffusion coefficient using Eq. (13) is shown in the inset of Fig. 2. The decrease in diffusion coefficient of nanoparticles in the presence of lysozyme could be because of an increase in protein-induced attractive interaction between nanoparticles and/or due to nanoparticle aggregation [45]. The sizes of the nanoparticles in these systems have been calculated using the Stokes-Einstein relation [Eq. (14)]. The size distributions of nanoparticles at different lysozyme concentrations are plotted in Fig. 3. The pure silica nanoparticles have an average

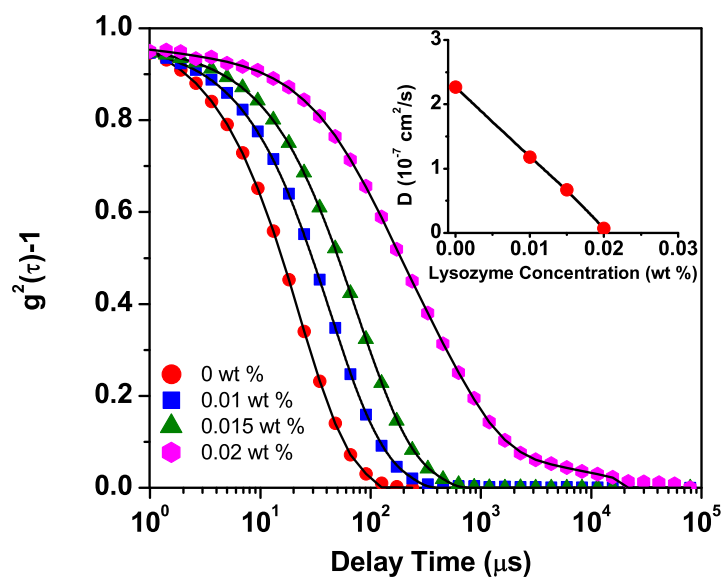

FIG. 2. (Color online) DLS data of 1 wt. \% HS40 silica nanoparticles with varying concentration of lysozyme protein in the presence of $0.1 M \mathrm{NaCl}$. Inset shows the variation of diffusion coefficient $(D)$ of nanoparticles with lysozyme concentration. 


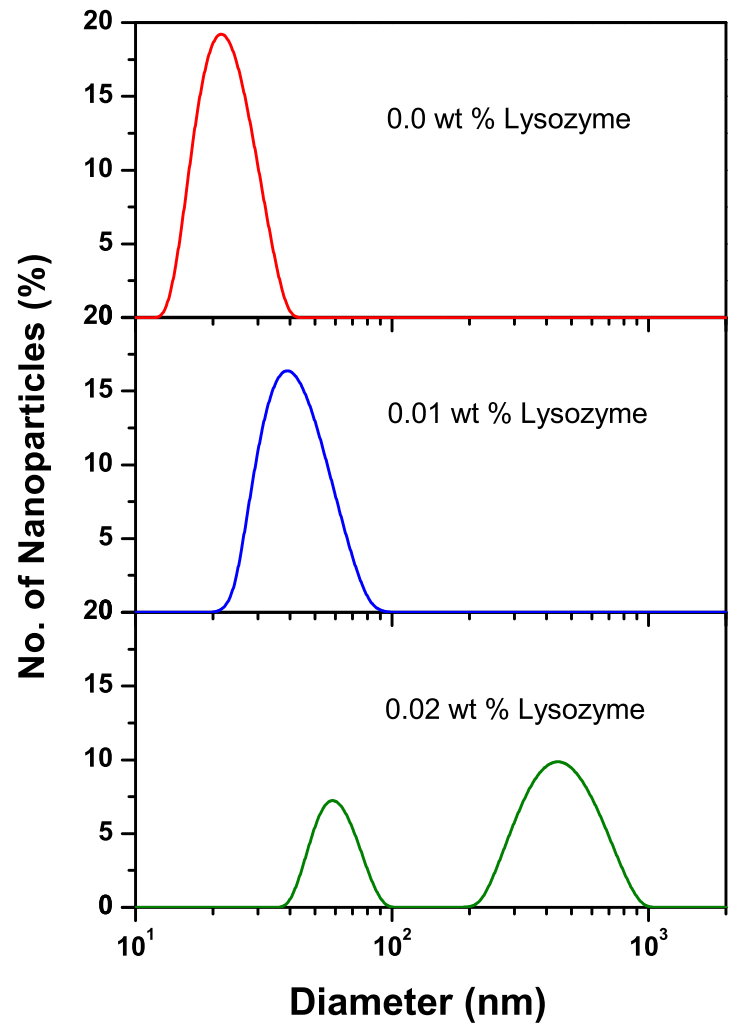

FIG. 3. (Color online) Calculated size distributions of nanoparticles from DLS data of $1 \mathrm{wt}$ \% HS40 silica nanoparticles with varying concentration of lysozyme protein in the presence of $0.1 \mathrm{M} \mathrm{NaCl}$.

hydrodynamic size (radius) of $10.5 \mathrm{~nm}(\mathrm{PDI}=0.2)$, which is in good agreement with those reported earlier [46]. There are two important features observed: (i) the average size of the nanoparticles increasing with lysozyme concentration and (ii) the appearance of a second size distribution (for higher lysozyme concentration) at much higher sizes than that of the first size distribution. The increase in the average size of nanoparticles of the first size distribution can be explained by enhancement in attractive interaction between nanoparticles and/or being due to emergence of the process of aggregation [47], whereas the second size distribution corresponds to the large nanoparticle aggregates representing a two-phase system. This phase behavior of silica nanoparticles with oppositely charged lysozyme protein arises because of the strong electrostatic binding of the protein with the nanoparticles $[19,23]$.

Despite the fact that silica nanoparticles and BSA protein have a similar charge (both are anionic), it has been seen in Fig. 1 that BSA can render in the similar phase behavior to that of nanoparticles with lysozyme. DLS data of a silica nanoparticle-BSA system are shown in Fig. 4. The features of the data are identical to that of a nanoparticle-lysozyme system. The size distributions in the nanoparticle-BSA system for different concentrations of BSA are plotted in Fig. 5. There is a possibility of site-specific adsorption of BSA on individual nanoparticles, which in turn could be the reason for the increase in the apparent size of the nanoparticle-protein complex. The sum of the hydrodynamic size of the nanoparticles $(10.5 \mathrm{~nm})$ and BSA $(7 \mathrm{~nm})$ is, however, much smaller than that measured

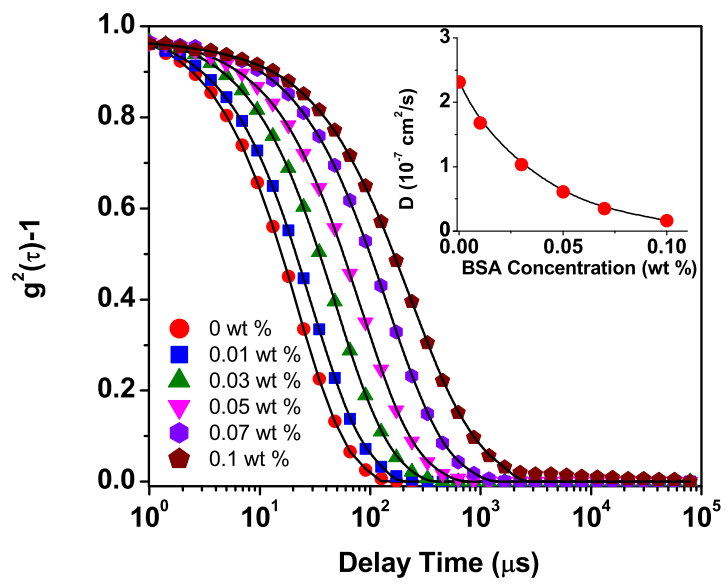

FIG. 4. (Color online) DLS data of 1 wt. \% HS40 silica nanoparticles with varying concentration of BSA protein in the presence of $0.1 \mathrm{M} \mathrm{NaCl}$. Inset shows the variation of diffusion coefficient $(D)$ of nanoparticles with BSA concentration.

in DLS experiments. Moreover, because both nanoparticle and protein are similarly charged, their complex will have higher surface charge density and hence cannot lead to any protein-mediated nanoparticle aggregation [21]. We believe that the changes in the DLS data (Fig. 4) are due to increase in the attractive interaction between nanoparticles. This can be explained by the nonadsorption of BSA protein, possibly leading to depletion interaction between nanoparticles [23]. The large nanoparticle aggregates (two-phase system) is achieved

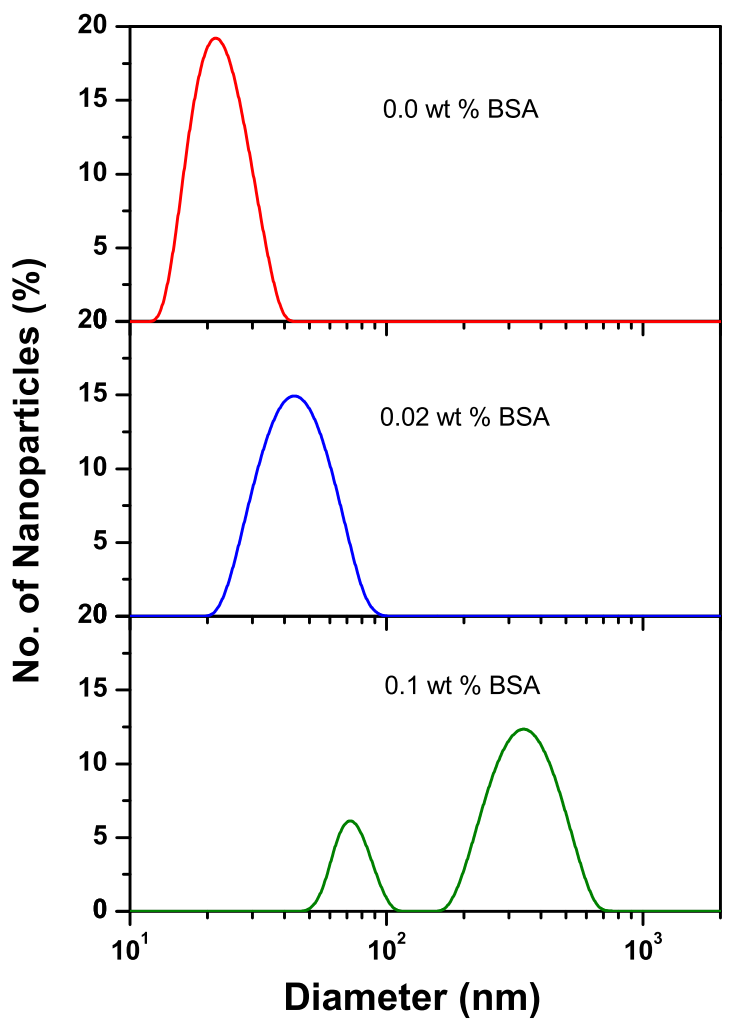

FIG. 5. (Color online) Calculated size distributions of nanoparticles from DLS data of $1 \mathrm{wt}$ \% HS40 silica nanoparticles with varying concentration of BSA protein in the presence of $0.1 \mathrm{M} \mathrm{NaCl}$. 

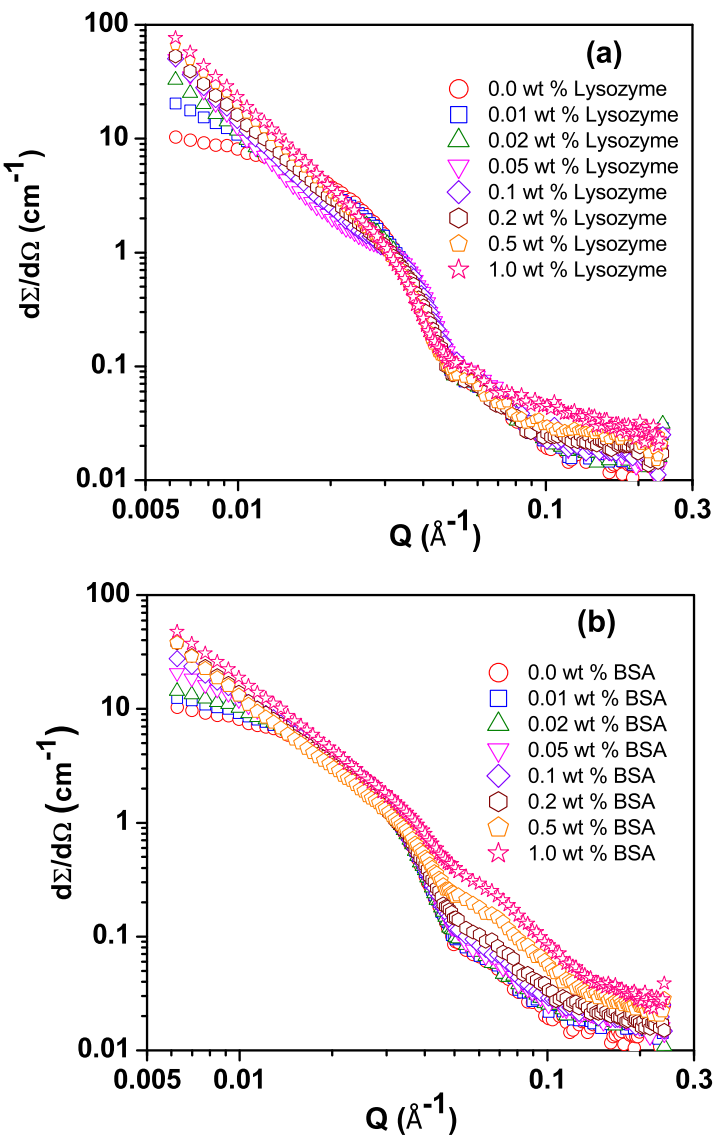

FIG. 6. (Color online) SANS data of 1 wt. \% HS40 silica nanoparticles in the presence of $0.1 \mathrm{M} \mathrm{NaCl}$ with varying concentrations of (a) lysozyme and (b) BSA proteins.

at higher BSA concentrations (Fig. 5) when the depletion interaction overcomes the electrostatic repulsion between nanoparticles. The modeling of these different interactions has been further investigated by SANS.

Figure 6 presents the SANS data of 1 wt. \% HS40 silica nanoparticles with varying concentrations of (a) lysozyme and (b) BSA proteins in the presence of $0.1 \mathrm{M} \mathrm{NaCl}$. The data are taken over a wide concentration range $(0-1$ wt. \%) for both the proteins to cover different regions of the phase behavior in Fig. 1. It is found that irrespective of the type of protein there is scattering buildup in the low- $Q$ regime with the increase in protein concentration. These data basically correspond to a region of phase behavior where nanoparticle-protein systems transform from a one-phase to a two-phase system. The data in the low- $Q$ regime do not show any change after some amount of protein concentration in the two-phase region, whereas there is an increase in the scattering in the higher- $Q$ regime as expected from the protein. Based on the overall features of the SANS data, the analysis has been divided into two sets of data where nanoparticle-protein systems (i) transform from a one-phase to a two-phase system and (ii) they have been fully transformed into a two-phase system.

The buildup of scattering with the increase in lysozyme concentration in the low- $Q$ regime of SANS data are fitted with two populations (nonaggregated and aggregated) of nanoparticles [Fig. 7(a)]. The oppositely charged lysozyme adsorbs
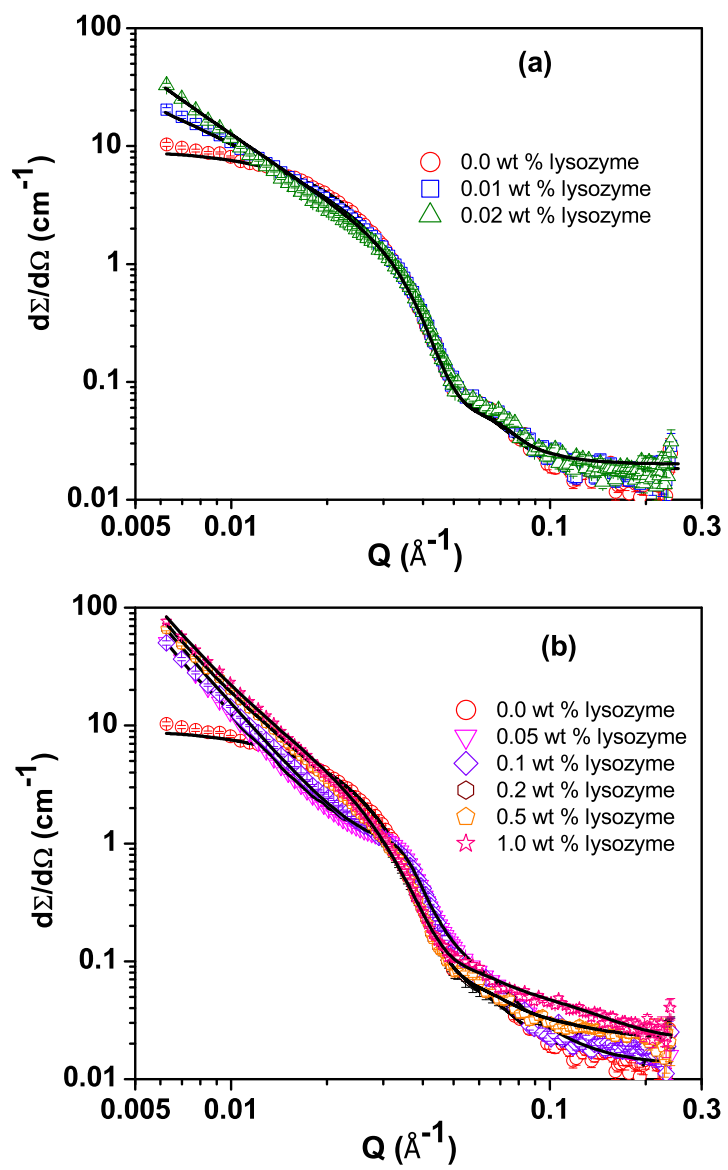

FIG. 7. (Color online) Fitted SANS data of 1 wt. \% HS40 silica nanoparticles with varying concentration of lysozyme protein in the presence of $0.1 \mathrm{M} \mathrm{NaCl}$. The data are divided into two sets of (a) low lysozyme concentration and (b) high lysozyme concentration.

on nanoparticles and results in protein-mediated aggregation of nanoparticles; the amount of aggregation depends on the protein concentration $[18,48]$. The aggregation phenomenon is modeled through the structure factor of $2 \mathrm{Y}$ potential between nonaggregated nanoparticles. The form factor for these nanoparticles used is that of polydispersed spherical. The aggregated nanoparticles are fitted with the fractal structures. The fitted parameters are given in Table I. The mean radius of nanoparticles is found to be $8.8 \mathrm{~nm}$ with a polydispersity of 0.2 [23]. The nanoparticle aggregates at low protein concentration are characterized by a surface fractal whose number fraction increases with the protein concentration. The $2 Y$ potential comprises four fitting parameters $K_{1}, K_{2}, Z_{1}$, and $Z_{2}$. The parameters $K_{1}$ and $Z_{1}$ represent the attractive part of the total potential to decide the strength (proportional to $K_{1}$ ) and range (proportional to $1 / Z_{1}$ ), respectively. On the other hand, $K_{2}$ and $Z_{2}$ provide the strength (related to effective charge) and range (related to ionic strength) of repulsive electrostatic interaction, respectively. The values of $K_{2}$ and $Z_{2}$ have been determined from the concentration-dependent SANS data of pure silica nanoparticles [23]. The effect of the electrolyte on these parameters is incorporated by Debye-Hückel theory as governed by the ionic strength of the system [49]. To minimize the number of fitting parameters during the data analysis, the values of $K_{2}$ and $Z_{2}$ were kept 
TABLE I. Fitted parameters of 1 wt. \% HS40 silica nanoparticles with varying concentration of lysozyme protein in the presence of $0.1 M \mathrm{NaCl}$.

(a) Low lysozyme concentration where the interacting nanoparticles coexist with nanoparticle aggregates. The data are fitted with $2 \mathrm{Y}$ potential. The parameters of repulsive interaction $K_{2}=1.5, Z_{2}=19.5$ are fixed.

\begin{tabular}{lccc} 
Lysozyme concentration $C($ wt. \%) & $K_{1}$ & $Z_{1}$ & $\begin{array}{c}\text { Fraction of aggregated } \\
\text { nanoparticles } \phi_{\mathrm{a}}(\%)\end{array}$ \\
\hline 0.01 & 12.5 & 9.0 & 17 \\
0.02 & 15.0 & 9.0 & 40
\end{tabular}

(b) Intermediate lysozyme concentration where the nanoparticle aggregates are characterized by a surface fractal.

\begin{tabular}{lcc}
\hline Lysozyme concentration $C($ wt. $\%)$ & Surface fractal dimension $D_{s}$ & $\begin{array}{c}\text { Volume fraction of nanoparticles } \\
\text { within the aggregates } \phi\end{array}$ \\
\hline 0.05 & 2.9 & 0.29 \\
0.1 & 2.8 & 0.23 \\
0.2 & 2.6 & 0.07 \\
\hline
\end{tabular}

(c) High lysozyme concentration where the nanoparticle aggregates are characterized by a mass fractal.

\begin{tabular}{lcc}
\hline Lysozyme concentration $C$ (wt. \%) & Mass fractal dimension $D_{m}$ & Building block radius $R_{\mathrm{b}}(\AA)$ \\
\hline 0.5 & 2.5 & 94.5 \\
1 & 2.5 & 95.0
\end{tabular}

fixed whereas the parameters of the attractive interaction $\left(K_{1}\right.$ and $Z_{1}$ ) were used as fitted parameters. It is found that the strength of attractive interaction is prominently high compared to the repulsive interaction. The range is of the order of the size of the lysozyme protein as protein mediating the attractive interaction between nanoparticles. The contribution of aggregated nanoparticles is considered as a surface fractal.

The SANS data corresponding to a two-phase system (nanoparticle aggregation) at higher lysozyme concentrations are shown in Fig. 7(b). All the scattering profiles show the linearity in the low- $Q$ regime indicating the fractal nature of nanoparticle aggregates [50]. It is observed that there are two groups of data with different slopes in the low- $Q$ region. The first group of data has a slope of about 3.2 describing the surface fractal behavior of the nanoparticle aggregates. The Bragg peak kind of feature around $Q_{p}=0.035 \AA^{-1}$ in these data indicates the ordered packing of nanoparticles within the aggregates. The sum of power law behavior for a surface fractal and contribution from ordered particles within the aggregate is used to fit the data [51]. The surface fractal dimension and the volume fraction of particles within the aggregates decrease with increasing lysozyme concentration. This is perhaps because of the competition of nanoparticleprotein (attractive) and protein-protein (repulsive) interactions resulting in change of the morphology of the nanoparticle aggregates. In the second group of data at higher lysozyme concentrations, the slope of the scattering curve decreases to a value about 2.5, suggesting morphology of nanoparticle aggregates changing from surface to mass fractal. This can be understood in terms of lysozyme molecules in a corona around the nanoparticle controlling the binding of nanoparticles.

The features of SANS data of silica nanoparticles with BSA [Fig. 6(b)] are quite similar to that of the silica nanoparticles with lysozyme protein [Fig. 6(a)]. The data are again divided into two sets corresponding to the regions of a one-phase to a two-phase system and only a two-phase system exists.
In the first set at low protein concentrations [Fig. 8(a)], the data are fitted with the interaction between nanoparticles via the $2 Y$ potential accounting for electrostatic repulsion and depletion attraction as induced by the nonadsorption of BSA. The fitted parameters are given in Table II. The parameters of electrostatic repulsion are kept fixed as used in the case of the nanoparticle-lysozyme system. Unlike the case of the nanoparticle-lysozyme system, the attractive depletion interaction for the nanoparticle-BSA system is found to be long range. Both the magnitude and range of this attractive interaction increase with the BSA concentration. The similar long-range attractive potential has also been reported in other systems [22,52]. The long-range attraction is justified from the fact that this interaction can induce aggregation in long-range repulsive charged stabilized systems. A fraction of aggregated nanoparticles also coexists in these systems. The fraction of aggregated nanoparticles increases with the BSA concentration. The contribution of aggregated nanoparticles is fitted as a mass fractal. In the second set of SANS data at high protein concentrations [Fig. 8(b)], the nanoparticle-BSA protein has mostly nanoparticle aggregates as characterized by a mass fractal. The differences in evolution of fractal structures of nanoparticle-BSA (mass fractal only) and nanoparticlelysozyme (surface to mass fractal) systems perhaps arise because of the different mechanisms of the aggregation for two systems.

The electrolyte effect with respect to the case without any electrolyte for nanoparticle-protein systems is compared in Fig. 9. In the case of the nanoparticle-lysozyme system, the comparison of SANS data with and without electrolyte for 1 wt. \% HS40 with 0.02 wt. \% lysozyme is shown in Fig. 9(a). The differences in the data, in particular in the low- $Q$ regime with and without electrolyte, arise as a result of the modifications in the interaction and structure of the system. Table III(a) gives the comparison of the fitted parameters in these systems. The parameters of repulsive interaction $\left(K_{2}\right.$ and $\left.Z_{2}\right)$ are kept constant and modified with electrolyte as decided by the 

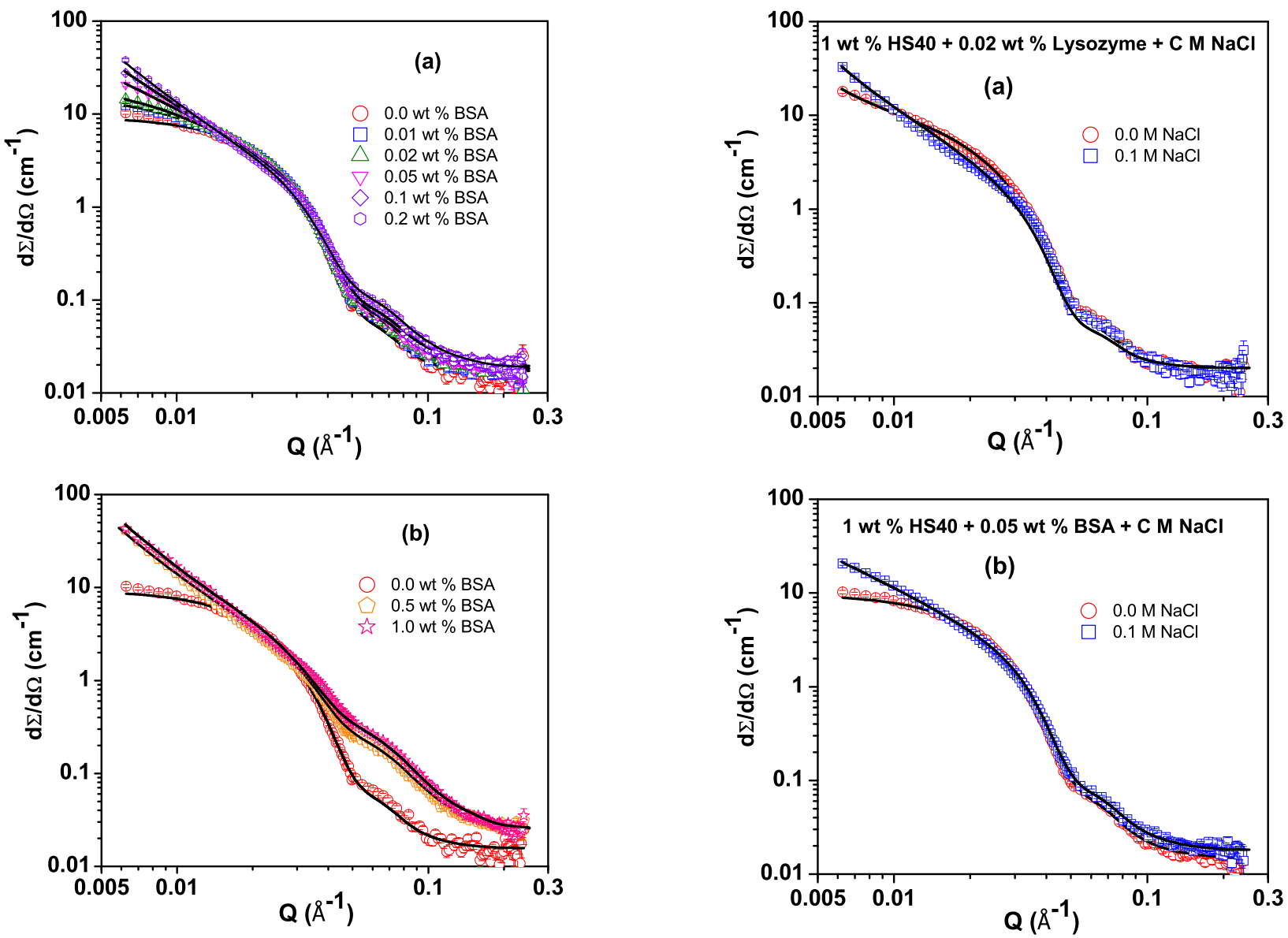

FIG. 8. (Color online) Fitted SANS data of 1 wt. \% HS40 silica nanoparticles with varying concentration of BSA protein in the presence of $0.1 \mathrm{M} \mathrm{NaCl}$. The data are divided into two sets of (a) low BSA concentration and (b) high BSA concentration.

change in the ionic strength of the system. Both the strength (proportional to $K_{2}$ ) and range (proportional to $1 / Z_{2}$ ) of the repulsive interaction as expected decrease with the electrolyte. The parameters of the lysozyme-mediated attractive interaction $\left(K_{1}\right.$ and $\left.Z_{1}\right)$ vary as $K_{1}$ deceases whereas $Z_{1}$ remains almost the same. Because this interaction is derived from the electrostatic nature, $K_{1}$ decreases in the presence of the electrolyte. The range of the interaction $\left(1 / Z_{1}\right)$ depends on the

FIG. 9. (Color online) Comparison of SANS data without and with $0.1 \mathrm{M} \mathrm{NaCl}$ : (a) 1 wt. $\%$ HS40 silica nanoparticles with 0.02 wt. \% lysozyme protein and (b) 1 wt. \% HS40 silica nanoparticles with 0.05 wt. \% BSA protein.

protein conformation, which is usually known to be unchanged at the moderate amount of electrolyte as is the present case. The total potential between nanoparticles and its individual components are plotted in Fig. 10(a). These observations suggest that the electrolyte effect in enhancing the nanoparticle aggregation of a silica nanoparticle-lysozyme system is primarily dictated by the decrease in the strength and range of the electrostatic repulsive interaction between nanoparticles.

TABLE II. Fitted parameters of 1 wt. \% HS40 silica nanoparticles with varying concentration of BSA protein in the presence of $0.1 M$ NaCl.

(a) Low BSA concentration where the interacting nanoparticles coexist with nanoparticle aggregates. The data are fitted with $2 \mathrm{Y}$ potential. The parameters of repulsive interaction $K_{2}=1.5, Z_{2}=19.5$ are fixed.

\begin{tabular}{lccc}
\hline BSA concentration $C$ (wt. \%) & $K_{1}$ & $Z_{1}$ & Fraction of aggregated nanoparticles $\phi_{\mathrm{a}}(\%)$ \\
\hline 0.01 & 18.0 & 5.5 & 0 \\
0.02 & 21.5 & 5.0 & 0 \\
0.05 & 24.0 & 4.8 & 15 \\
0.1 & 27.5 & 4.5 & 30 \\
0.2 & 32.0 & 4.2 & 60
\end{tabular}

(b) High BSA concentration where the nanoparticle aggregates are characterized by a mass fractal.

\begin{tabular}{lcc}
\hline BSA concentration $C$ (wt. \%) & Mass fractal dimension $D_{m}$ & Building block radius $R_{\mathrm{b}}(\AA)$ \\
\hline 0.5 & 2.5 & 90.5 \\
1 & 2.5 & 91.0 \\
\hline \hline
\end{tabular}


TABLE III. Comparison of fitted parameters of $2 \mathrm{Y}$ interaction potential without and with $0.1 \mathrm{M} \mathrm{NaCl}$.

(a) 1 wt. \% HS40 silica nanoparticles with 0.02 wt. \% lysozyme protein

\begin{tabular}{lcccr}
\hline$[\mathrm{NaCl}]$ & $K_{1}$ & $Z_{1}$ & $K_{2}$ & $Z_{2}$ \\
\hline $0 M$ & 20.0 & 9.0 & 9.0 & 7.0 \\
$0.1 M$ & 15.0 & 9.0 & 1.5 & 19.5
\end{tabular}

(b) 1 wt. \% HS40 silica nanoparticles with 0.05 wt. \% BSA protein

\begin{tabular}{lllll}
\hline $\mathrm{NaCl}]$ & $K_{1}$ & $Z_{1}$ & $K_{2}$ & $Z_{2}$ \\
\hline $0 M$
\end{tabular}

\begin{tabular}{lllll}
\hline $0 M$ & 7.5 & 4.8 & 9.0 & 7.0
\end{tabular}

$\begin{array}{lllll}0.1 M & 24.0 & 4.8 & 1.5 & 19.5\end{array}$

Figure 9(b) compares the nanoparticle-BSA system with and without electrolyte for $1 \mathrm{wt}$. \% HS40 with $0.05 \mathrm{wt}$ \% BSA. The changes in features of the data of a nanoparticle-BSA system are observed to be different than that of a nanoparticlelysozyme system. The comparison of the fitted parameters in a nanoparticle-BSA system without and with electrolyte is given in Table III(b). The electrostatic repulsive interaction has the same variation as in the case of lysozyme [Table III(a)]. The parameters of the BSA-induced depletion interaction ( $K_{1}$ and $Z_{1}$ ) show a trend where $K_{1}$ increases (unlike the nanoparticle-lysozyme system) whereas $Z_{1}$ does not change. The increase in the strength of the depletion interaction (proportional to $K_{1}$ ) can be understood in terms of decrease in protein-protein repulsion in the presence of an electrolyte. The suppression of repulsion significantly increases the excluded volume available for proteins and hence higher magnitude of depletion interaction [22,51]. The total potential between nanoparticles and its electrostatic and depletion components are plotted in Fig. 10(b). The results show that the presence of an electrolyte leads in significant modifications in both the electrostatic and depletion interactions in a nanoparticle-BSA system. These modifications in interactions are responsible for the change in phase behavior of the nanoparticle-BSA system. The changes are found different for lysozyme and BSA proteins and hence differences in their phase behavior.

\section{CONCLUSIONS}

The phase behavior of silica nanoparticles with two globular proteins, lysozyme and BSA, in the presence of an electrolyte has been studied by SANS and DLS techniques. Both the proteins show a similar kind of phase behavior where the respective nanoparticle-protein system transforms from a onephase to a two-phase system. This transformation of phase is observed at a much lower concentration for lysozyme than BSA. The phase behavior for both the proteins is significantly modified in the presence of an electrolyte. The protein concentration required for phase transformation is suppressed
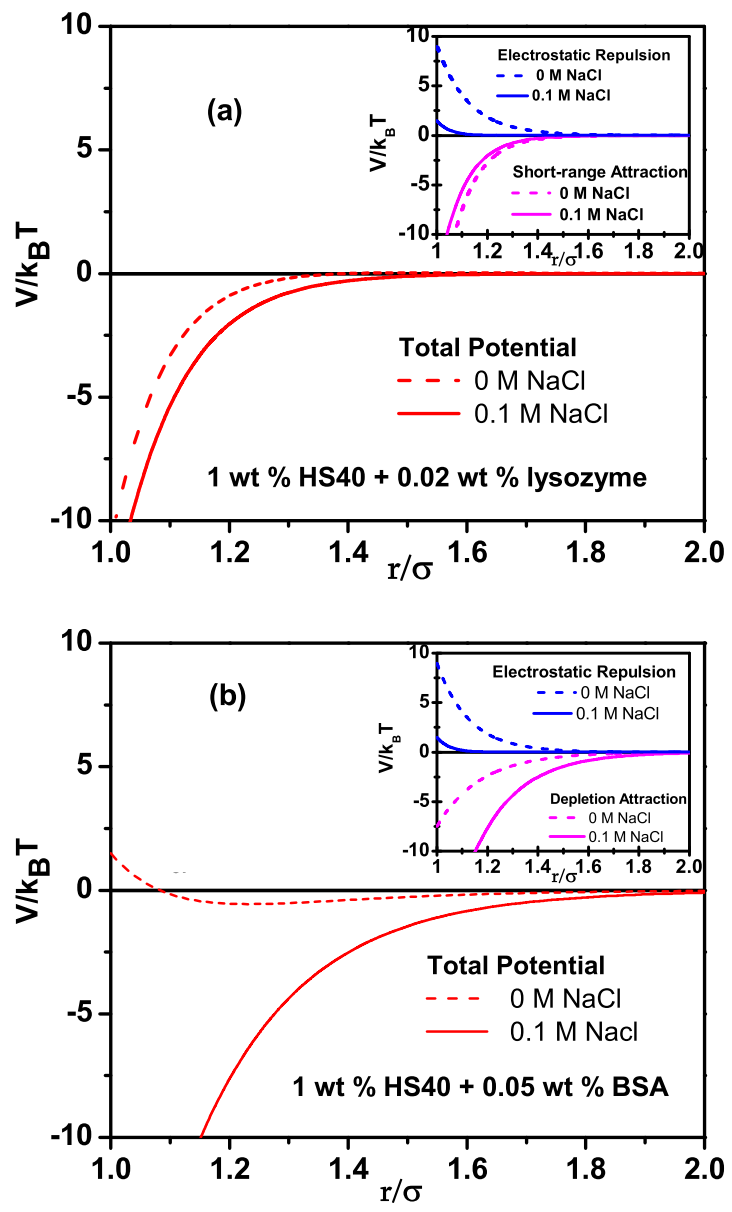

FIG. 10. (Color online) The calculated total interaction potential between nanoparticles without and with $0.1 \mathrm{M} \mathrm{NaCl}$ : (a) $1 \mathrm{wt}$. \% HS40 silica nanoparticles with $0.02 \mathrm{wt}$. \% lysozyme protein and (b) $1 \mathrm{wt}$ \% HS40 silica nanoparticles with 0.05 wt. \% BSA protein. Insets show the repulsive and attractive components of the total potential.

on addition of the electrolyte and the effect is more pronounced for BSA than lysozyme. SANS and DLS data suggest that changes in phase behavior are related with modifications in both interaction and structure of the nanoparticle-protein systems. The combination of short-range attraction and electrostatic repulsion for lysozyme protein leads to proteinmediated aggregation of nanoparticles, whereas nonadsorption of BSA protein with nanoparticles induces long-range depletion interaction to overcome electrostatic repulsion to aggregate nanoparticles. The suppression of the electrostatic repulsion for lysozyme protein and enhancement of depletion interaction for BSA are found to be mainly responsible for the changes in the phase behavior of respective nanoparticleprotein systems in the presence of an electrolyte.
[1] R. Langer and D. A. Tirrell, Nature (London) 428, 487 (2004).

[2] M. Stodulski and T. Gulder, Angew. Chem., Int. Ed. 51, 11202 (2012).
[3] M. Mahmoudi, I. Lynch, M. R. Ejtehadi, M. P. Monopoli, F. B. Bombelli, and S. Laurent, Chem. Rev. 111, 5610 (2011).

[4] I. Lynch and K. A. Dawson, Nano Today 3, 40 (2008). 
[5] M.-E. Aubin-Tam and K. Hamad-Schifferli, Biomed. Mater. 3, 034001 (2008).

[6] M. Bruchez, Jr., M. Moronne, P. Gin, S. Weiss, and A. P. Alivisatos, Science 281, 2013 (1998).

[7] A. E. Nel, L. Mädler, D. Velegol, T. Xia, E. M. V. Hoek, P. Somasundaran, F. Klaessig, V. Castranova, and M. Thompson, Nat. Mater. 8, 543 (2009).

[8] T. Zhu, Z. Jiang, and Y. Ma, Appl. Phys. Lett. 102, 153109 (2013).

[9] S. R. Saptarshi, A. Duschl, and A. L. Lopata, J. Nanobiotechnol. 11, 26 (2013).

[10] M. Lundqvist, I. Sethson, and B. H. Jonsson, Langmuir 20, 10639 (2004).

[11] Y. Min, M. Akbulut, K. Kristiansen, Y. Golan, and J. Israelachvili, Nat. Mater. 7, 527 (2008).

[12] H. Yuan, J. Li, G. Bao, and S. Zhang, Phys. Rev. Lett. 105, 138101 (2010).

[13] T. Missana and A. Adell, J. Colloid Interface Sci. 230, 150 (2000).

[14] L. Belloni, J. Phys.: Condens. Matter 12, R549 (2000).

[15] M. Boström, D. R. M. Williams, and B. W. Ninham, Phys. Rev. Lett. 87, 168103 (2001).

[16] M. Ruths, H. Yoshizawa, L. J. Fetters, and J. N. Israelachvili, Macromolecules 29, 7193 (1996).

[17] K. Rezwan, L. P. Meier, and L. J. Gauckler, Biomaterials 26, 4351 (2005).

[18] S. Kumar, V. K. Aswal, and J. Kohlbrecher, Langmuir 27, 10167 (2011).

[19] B. Bharti, J. Meissner, S. H. L. Klapp, and G. H. Findenegg, Soft Matter 10, 718 (2014).

[20] T. J. Su, J. R. Lu, R. K. Thomas, Z. F. Cui, and J. Penfold, J. Phys. Chem. B 102, 8100 (1998).

[21] J. Wang, U. B. Jensen, G. V. Jensen, S. Shipovskov, V. S. Balakrishnan, D. Otzen, J. S. Pedersen, F. Besenbacher, and D. S. Sutherland, Nano Lett. 11, 4985 (2011).

[22] S. Kumar, M.-J. Lee, V. K. Aswal, and S.-M. Choi, Phys. Rev. E 87, 042315 (2013).

[23] I. Yadav, S. Kumar, V. K. Aswal, and J. Kohlbrecher, Phys. Rev. E 89, 032304 (2014).

[24] D. I. Svergun and M. H. J. Koch, Rep. Prog. Phys. 66, 1735 (2003).

[25] G. D. Wignall and Y. B. Melnichenko, Rep. Prog. Phys. 68, 1761 (2005).

[26] J. S. Pedersen, Adv. Colloid Interface Sci. 70, 171 (1997).

[27] Y. Liu, W.-R. Chen, and S.-H. Chen, J. Chem. Phys. 122, 044507 (2005).

[28] Y. Liu, E. Fratini, P. Baglioni, W.-R. Chen, and S.-H. Chen, Phys. Rev. Lett. 95, 118102 (2005).
[29] H. Jans, X. Liu, L. Austin, G. Maes, and Q. Huo, Anal. Chem. 81, 9425 (2009).

[30] D. Kleshchanok, M. Heinen, G. Nagele, and P. Holmqvist, Soft Matter 8, 1584 (2012).

[31] J. Kohlbrecher and W. Wagner, J. Appl. Crystallogr. 33, 804 (2000).

[32] S. H. Chen, E. Y. Sheu, J. Kalus, and H. Hoffmann, J. Appl. Crystallogr. 21, 751 (1988).

[33] J. B. Hayter and J. Penfold, Colloid Polym. Sci. 261, 1022 (1983).

[34] C. G. Windsor, J. Appl. Crystallogr. 21, 582 (1988).

[35] A. J. Chinchalikar, V. K. Aswal, J. Kohlbrecher, and A. G. Wagh, Phys. Rev. E 87, 062708 (2013).

[36] A. Shukla, E. Mylonas, E. Di Cola, S. Finet, P. Timmins, T. Narayanan, and D. I. Svergun, Proc. Natl. Acad. Sci. USA 105, 5075 (2008).

[37] A. P. Radlinski, E. Z. Radlinska, M. Agamalian, G. D. Wignall, P. Lindner, and O. G. Randl, Phys. Rev. Lett. 82, 3078 (1999).

[38] D. F. R. Mildner and P. L. Hall, J. Phys. D: Appl. Phys. 19, 1535 (1986).

[39] J. Teixeira, J. Appl. Crystallogr. 21, 781 (1988).

[40] P. R. Bevington, Data Reduction and Error Analysis for Physical Sciences (McGraw-Hill, New York, 1969).

[41] R. Pecora, Dynamic Light Scattering (Plenum, New York, 1985).

[42] S. Chodankar, V. K. Aswal, J. Kohlbrecher, R. Vavrin, and A. G. Wagh, Phys. Rev. E 77, 031901 (2008).

[43] B. J. Frisken, Appl. Opt. 40, 4087 (2001).

[44] D. E. Koppel, J. Chem. Phys. 57, 4814 (1972).

[45] L. Porcar, P. Falus, W.-R. Chen, A. Faraone, E. Frantini, K. Honh, P. Baglioni, and Y. Liu, J. Phys. Chem. Lett. 1, 126 (2010).

[46] S. Kumar, V. K. Aswal, and J. Kohlbrecher, Langmuir 28, 9288 (2012).

[47] G. Orts-Gil, K. Natte, R. Thiermann, M. Girod, S. Rades, H. Kalbe, A. F. Thünemann, M. Maskos, and W. Österle, Colloids Surf. B 108, 110 (2013).

[48] B. Bharti, J. Meissner, and G. H. Findenegg, Langmuir 27, 9823 (2011).

[49] Y. Zeng, S. Grandner, C. L. P. Oliveira, A. F. Thunemann, O. Paris, J. S. Pedersen, S. H. L. Klapp, and R. von Klitzing, Soft Matter 7, 10899 (2011).

[50] D. Rojanski, D. Huppert, H. D. Bale, Xie Dacai, P. W. Schmidt, D. Farin, A. Seri-Levy, and D. Avnir, Phys. Rev. Lett. 56, 2505 (1986).

[51] S. Kumar, D. Ray, V. K. Aswal, and J. Kohlbrecher, Phys. Rev. E 90, 042316 (2014).

[52] J. C. Crocker, J. A. Matteo, A. D. Dinsmore, and A. G. Yodh, Phys. Rev. Lett. 82, 4352 (1999). 